\title{
Análisis de la topografía lesional en la baja de combate. Experiencia de la Sanidad Militar española desplegada en Herat (Afganistán)
}

\author{
Navarro Suay R. ${ }^{1}$, Hernández-Abadía de Barbará A. ${ }^{2}$, Gutiérrez Ortega C. ${ }^{3}$, Bartolomé Cela E. ${ }^{4}$, \\ Tamburri Bariain R. ${ }^{1}$, Gilsanz Rodríguez F. ${ }^{5}$
}

Sanid. mil. 2011; 67 (2): 78-83; ISSN: 1887-8571

\begin{abstract}
RESUMEN
Introducción: La localización corporal de las lesiones por arma de fuego y por artefactos explosivos en el combatiente ha seguido un patrón diferente a lo largo de la historia. La distribución anatómica de dichas lesiones en los últimos conflictos ha demostrado que las áreas más afectadas son las extremidades, seguidas de la cabeza y el cuello. El objetivo del estudio es describir la experiencia de Médicos Militares españoles desplegados en el Hospital Militar Role 2 de Herat (Afganistán) sobre las regiones corporales lesionadas de las bajas de combate de 2005 a 2008. Material y Método: Se realizó un estudio descriptivo, transversal sobre todo el personal civil y militar, que hubiese recibido herida por arma de fuego o por artefacto explosivo en la Región Oeste de Afganistán y fuese atendido por el ROLE 2 español de Herat (Afganistán) durante los años 2005-2008. Resultados: El número total de casos analizados fue de 256. El área topográfica más afectada en las bajas analizadas fue miembros inferiores $(n=123,48 \%)$, seguido de miembros superiores $(n=99,39 \%)$ y abdomen $(n=56,22 \%)$. El área anatómica donde los explosivos $(\mathrm{n}=85)$ y las armas de fuego $(\mathrm{n}=38)$ han producido más lesiones ha sido los miembros inferiores. Algo más de la mitad de las bajas atendidas $(\mathrm{n}=142,55 \%)$ presentaron una única región afectada: $59(23 \%)$. Conclusiones: En nuestro estudio, las áreas anatómicas más afectadas tanto por explosivo como por arma de fuego han sido los miembros inferiores y miembros superiores. Este hecho coincide con los datos obtenidos en otras series.
\end{abstract}

PALABRAS CLAVE: Anatomía, Arma de fuego, Explosivo, Afganistán, Sanidad Militar española.

\section{Analysis of the lesional topography in the combat casualty. The Spanish Medical Service experience in Herat (Afghanistan).} SUMMARY

Introduction: Along the history the localization in the combatant's body of the wounds due to firearms and explosive artifacts has followed different patterns. The anatomical distribution of these injuries in the last conflicts has proven that the most affected areas are the limbs followed by the head and neck. The objective of this study is to describe the experience of the Spanish medical officers deployed in the military hospital in Herat (Afghanistan) about the body parts injured in combat casualties from 2005 to 2008. Materials and Methods: A descriptive, transversal study was carried out including all civilian and military personnel wounded by explosive artifacts or firearms in the West Region of Afghanistan and taken care of in the Spanish Role 2 from 2005 to 2008. Results: The total number of cases analyzed was 256. In the studied casualties the most affected area was the lower limbs ( $\mathrm{n}=123 ; 48 \%)$, followed by the upper limbs ( $\mathrm{n}=99 ; 39 \%)$ and abdomen $(n=56 ; 22 \%)$. The anatomical area where explosives $(n=85)$ and firearms $(n=38)$ have caused more injuries is the lower limbs. A little more than half the casualties ( $\mathrm{n}=142 ; 55 \%)$ presented only one affected area: 59 (23\%). Conclusions: In our study the lower and upper limbs have been the anatomical areas most affected by explosives as well as firearms. These data coincides with the results obtained in other series.

KEY WORDS: Anatomy, Firearm, Explosive, Afghanistan, Spanish Medical Service.

\section{INTRODUCCION}

La historia de la humanidad está tristemente marcada por gran cantidad de conflictos armados. Una consecuencia de este hecho, es que el cuidado al herido en los campos de batalla exista desde que el hombre ha comenzado a agredirse entre sí.

${ }^{1}$ Cap. Médico. Escuela Militar de Sanidad. Madrid. España.

${ }^{2}$ Cte. Médico. Hospital Central de la Defensa Gómez Ulla. Servicio de Telemedicina. Madrid. España.

${ }^{3}$ Hospital Central de la Defensa Gómez Ulla. Servicio de Calidad y Medicina Preventiva. Madrid. España.

${ }^{4}$ Tcol. Médico. Hospital Central de la Defensa «Gómez Ulla». Unidad de Cuidados Intensivos. Madrid. España.

${ }_{5}^{5}$ Universidad Autónoma de Madrid. Departamento de Cirugía. H.U. La Paz. Servicio de Anestesiología y Reanimación. Madrid. España.

Dirección para correspondencia: r_navarro_suay@yahoo.es.

Recibido: 16 de febrero de 2010

Aceptado: 29 de junio de 2010
La localización corporal de las lesiones por arma de fuego y por artefactos explosivos en el combatiente ha seguido un patrón diferente a lo largo de los conflictos, ya que influyen aspectos muy dispares, como son el tipo de arma empleada, la protección conseguida, ambiente, la situación táctica ${ }^{1} \ldots$

La distribución anatómica de las lesiones penetrantes en los heridos de las principales guerras del siglo $\mathrm{XX}$ ha demostrado que las áreas más afectadas son las extremidades, seguidas de la cabeza y el cuello. Asimismo, se ha observado cómo los artefactos explosivos y las armas de fuego, son los principales agentes lesionantes en los conflictos más recientes ${ }^{2}$.

El objetivo del estudio es describir la experiencia de Médicos Militares españoles desplegados en el Hospital Militar de Herat (Afganistán) sobre las regiones corporales lesionadas de las bajas de combate de 2005 a 2008. 


\section{MATERIAL Y MÉTODO}

Se ha diseñado un estudio descriptivo, transversal, sobre todo el personal civil y militar, que hubiese recibido herida por arma de fuego o por artefacto explosivo en la Región Oeste de Afganistán y fuese atendido por el ROLE 2 español de Herat (Afganistán) durante los años 2005-2008, empleándose como criterio de inclusión todas aquellas personas que hubiesen sufrido una herida de arma de fuego o lesiones por artefacto explosivo tanto sobre el terreno como durante un vuelo. No se emplearon criterios de exclusión.

Las variables incluidas en el estudio fueron independientes (sociodemográficas y de control: sexo, edad, civil/militar, zona geográfica de procedencia, transporte sanitario) y dependientes (área anatómica -politómica: cabeza-cuello, tórax, abdomen, miembros superiores, miembros inferiores-).

Para la realización del estudio se revisaron 12.256 historias clínicas, y la recopilación de los datos se llevó a cabo mediante una ficha de recogida de datos realizada para este trabajo.

El método estadístico empleado fue el descriptivo. Para variables categóricas se emplearon sus frecuencias absolutas y relativas en tantos por ciento (\%).

\section{RESULTADOS}

Durante el periodo del estudio, se recopilaron un total de 256 bajas que habían sufrido lesiones diversas producidas durante enfrentamientos armados.

La mayoría de las bajas fueron varones ( $\mathrm{n}=246,96 \%$ ), únicamente $(n=10,4 \%)$ eran mujeres. Casi la mitad de las bajas $(n=101$, $39 \%$ ) tenían entre 25 y 29 años. El grupo con más bajas atendidas ha sido el ANA (Afghan National Army) $(n=112,44 \%)$. Las provincias desde donde se evacuaron más heridos han sido Farah, en el Sur ( $n=134,53 \%)$ y Badghis, en el Norte $(n=54,21 \%)$. El medio de evacuación más empleado ha sido MEDEVAC (aeroevacuación medicalizada) ( $\mathrm{n}=194,76 \%)$.

El área topográfica más afectada en las bajas analizadas fue miembros inferiores ( $\mathrm{n}=123,48 \%$ ), seguido de miembros superiores $(\mathrm{n}=99,39 \%)$ y abdomen $(\mathrm{n}=56,22 \%)$ (Figura 1$)$.

$\mathrm{El}$ área anatómica donde los explosivos $(\mathrm{n}=85)$ y las armas de fuego $(\mathrm{n}=38)$ han producido más lesiones ha sido los miembros inferiores.

En todas las divisiones anatómicas, el explosivo fue el agente causal de la mayor parte de las lesiones.

Las áreas topográficas en donde se han evidenciado una mayor diferencia proporcional entre las lesiones por explosivos y arma de fuego han sido cabeza y cuello.

Algo más de la mitad de las bajas atendidas ( $\mathrm{n}=142,55 \%)$ presentaron una única región afectada: 59 (23\%) padecieron heridas en 2 regiones, mientras que $39(15,5 \%)$ sufrieron lesiones en 3 áreas anatómicas (Figura 2).

La mayor parte de los heridos con dos o más áreas anatómicas dañadas recibieron los impactos de un explosivo.

\section{DISCUSIÓN}

Desde tiempos muy remotos, el hombre se enfrentó con guerras y catástrofes que provocaron múltiples lesiones traumáticas. Esta



Figura 1. Bajas por agente lesional y área anatómica lesionada.

situación generó el desafío de proporcionar a las víctimas tratamientos adecuados para su correcta recuperación. A este hecho se suma, que la responsabilidad médica durante los conflictos armados radica en estar preparados y adaptados al tipo de bajas que presumiblemente se tendrán que atender en este escenario. Por este motivo, desde nuestro punto de vista es interesante analizar las peculiaridades de las bajas de anteriores conflictos, para poder comprender de un modo más adecuado, las heridas que sufren los soldados durante las operaciones militares actuales y tomar las adecuadas medidas logístico-operativas y asistenciales para el futuro.

La primera referencia de esta necesidad nos la encontramos ya en el siglo VIII aC, concretamente en el Canto IX de la Iliada de Homero. Este autor considera que «un médico vale más que muchos soldados, ya que sabe arrancar flechas y aplicar medicinas calmantes a los heridos» ${ }^{3}$.

Además de valorar la necesidad del médico en el combate, Homero destaca la importancia de analizar las regiones lesionadas de los heridos durante la confrontación armada. De hecho, si analiza-

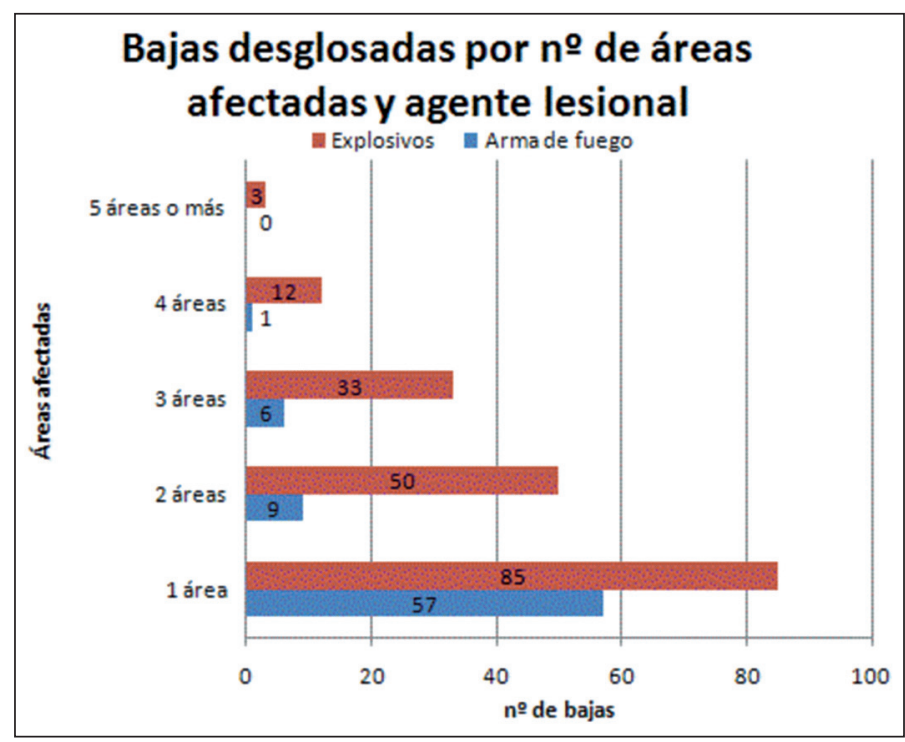

Figura 2. Bajas desglosadas por número de áreas afectadas y agente lesional. 
Tabla 1. Distribución anatómica de las lesiones penetrantes $(\%)^{6,7}$. No se contemplan las bajas totales de cada una de las guerras.

\begin{tabular}{|c|c|c|c|c|c|}
\hline Guerra & Cabeza/Cuello & Tórax & Abdomen & Miembros & Otros \\
\hline I Guerra Mundial (1914-18) & 17 & 4 & 2 & 70 & 7 \\
\hline II Guerra Mundial (1939-45) & 4 & 8 & 4 & 75 & 9 \\
\hline Guerra de Corea (1950-53) & 17 & 7 & 7 & 67 & 2 \\
\hline Guerra de Vietnam (1958-75) & 14 & 7 & 5 & 74 & \\
\hline Irlanda del Norte (1968-94) & 20 & 15 & 15 & 50 & \\
\hline Malvinas (1982) & 16 & 15 & 10 & 59 & \\
\hline Líbano (1982) & 11,5 & 16 & 18 & 45,5 & 9 \\
\hline Guerra del Golfo (GB) (1990-91) & 6 & 12 & 11 & 71 & $32 *$ \\
\hline Guerra del Golfo (EEUU) (1990-91) & 11 & 8 & 7 & 56 & $18 \dagger$ \\
\hline Somalia (1993) & 20 & 8 & 5 & 65 & $2 \dagger$ \\
\hline Afganistán (EEUU) $(2001-\ldots)$ & 16 & 12 & 11 & 61 & \\
\hline Chechenia (Rusia) (1999-02) & 24 & 9 & 4 & 63 & \\
\hline
\end{tabular}

* No están incluidos heridas en dorso, glúteos y múltiples por metralla.

$\dagger$ Lesiones múltiples.

mos La Iliada, podemos encontramos 159 lesiones traumáticas, de las cuales las localizaciones con mayor número de lesiones son la cabeza y cara, el tórax y el cuello ${ }^{4}$.

Cuatro siglos más tarde, aproximadamente 400 años a. C, Hipócrates en el Corpus Hipocraticum vuelve a valorar la presencia del médico durante el combate, al decir que «aquel que desee ser médico, debe alistarse al ejército y seguir sus pasos allá donde vaya. Es la forma de adquirir experiencia». Además añade que «conocer las lesiones que han dejado las armas que han penetrado en el cuerpo es una parte importantísima del arte de la cirugía. Solo aquel que es experto en estas lesiones puede intervenir de manera adecuada» ${ }^{5}$.

Si nos centramos en la distribución anatómica de las lesiones penetrantes en los heridos de los últimos conflictos armados en siglo $\mathrm{XX}$, podemos observar que por lo general, las áreas más afectadas son los miembros, seguidas de la cabeza y el cuello ${ }^{6,7}$ (Tabla 1).

A continuación, detallaremos la afectación de las distintas regiones corporales en las bajas durante varios conflictos armados.

\section{Guerra Mundial (1939-1945)}

En la Tabla 3 se muestra como dos tercios de las bajas norteamericanas durante este conflicto presentan lesiones en las extremidades ${ }^{8,9}$.

Tabla 2. Distribución de bajas norteamericanas por agentes penetrantes en la II Guerra Mundial ${ }^{8,9}$.

\begin{tabular}{|llll|}
\hline Región corporal & Previsto* & $\begin{array}{l}\text { Todas bajas } \\
\text { terrestres** } \\
\mathbf{( \% )}\end{array}$ & $\begin{array}{l}\text { Bajas que llegan } \\
\text { hasta Instalaciones } \\
\text { Sanitarias***(\%) }\end{array}$ \\
\hline Cabeza, Cara, Cuello & 12 & 21 & 15 \\
Tórax & 16 & 12 & 10 \\
Abdomen & 11 & 11,5 & 5 \\
Extremidad superior & 22 & 23,5 & 28 \\
Extremidad inferior & 39 & 35 & 41,5 \\
\hline
\end{tabular}

* Previsto: El área corporal se ha ajustado a las posturas que adopta el soldado (prono, de rodillas, bipedestacion) durante el combate.

** Todas bajas terrestres: Bajas al completo, tanto las fallecidas antes de llegar a una instalación sanitaria, como aquellas que consiguieron ser atendidas medicamente. *** Bajas que llegan hasta Instalaciones Sanitarias: Bajas que consiguen ser atendidas medicamente.
La distribución de lesiones en cabeza, cara y cuello prevista (primera columna de la Tabla 2) es considerablemente inferior a la del total de bajas terrestres (segunda columna de la Tabla 2) ) $^{8,9}$. Este incremento puede ser debido a que por razones tácticas, el soldado expone su cabeza más que el resto del cuerpo. Por tanto, tras analizar esta Tabla, podemos afirmar que la distribución de heridas sigue un patrón paralelo a la superficie corporal (aparecen más heridas en una determinada área topográfica cuanto mayor es la superficie corporal de la misma), a excepción de la región cefálica, que está afectada el doble de lo previsto.

El motivo más probable por el que únicamente consiguen llegar vivos a instalaciones sanitarias el $15 \%$ de las bajas con lesiones cefálicas, puede ser debido a la alta letalidad de las heridas localizadas en esta región anatómica.

\section{Guerra de Corea (1950-1953)}

La distribución anatómica de las heridas producidas en la Guerra de Corea queda recogida como sigue: miembros $61 \%$, tórax $16 \%$, cabeza $12 \%$ y abdomen $11 \%{ }^{10}$.

\section{Guerra de Vietnam (1965-1970)}

El estudio WDMET (Wound Data and Munitions Effectiveness Team $)^{11}$ describe 8.000 bajas del Ejército y del Cuerpo de Marines norteamericano durante 18 meses desde 1967 hasta 1969. Concluye que del total de las bajas que fallecieron, dos terceras partes fueron por heridas en cabeza y en tórax, y que las heridas en tejidos blandos y en extremidades afectaron a tres quintas partes de los supervivientes. Sus resultados globales se muestran en la Tabla 3.

\section{Guerra de las Malvinas (1982)}

La distribución de bajas argentinas y británicas atendidas en una instalación sanitaria del Ejército británico queda reflejada a continuación: cabeza y cuello $(\mathrm{n}=36 ; 14 \%)$, tórax $(\mathrm{n}=18 ; 7 \%)$, abdomen 
Tabla 3. Distribución de lesiones por área anatómica en heridos y fallecidos en la Guerra de Vietnam ${ }^{11}$.

\begin{tabular}{|lcll|}
\hline \multicolumn{1}{c}{ HERIDOS } & \multicolumn{2}{c|}{ FALLECIDOS } \\
\hline Área anatómica & $\%$ & Área anatómica & $\%$ \\
\hline Superficiales & 47 & Cabeza & 38 \\
Extremidades & 26 & Tórax & 24 \\
Abdomen & 8 & Múltiples & 17 \\
Múltiples & 5 & Abdomen & 9 \\
Cabeza & 4 & Cara & 4 \\
Cara & 4 & Cuello & 4 \\
Tórax & 4 & Extremidades & 3 \\
Cuello & 2 & Superficiales & 1 \\
\hline
\end{tabular}

Superficiales: Aquellas que afectan a piel, tejido celular subcutáneo y musculatura estriada Múltiples: Aquellas bajas cuyas heridas afectan a dos áreas corporales diferentes.

y pelvis $(n=30 ; 11,5 \%)$, miembro superior $(n=68 ; 26,5 \%)$ y miembro inferior $(\mathrm{n}=106 ; 41 \%)^{12}$.

\section{Guerra de Bosnia-Hercegovina (1992-1995)}

Según los estudios de Villanueva ${ }^{13}$ y Villalonga ${ }^{14}$, las regiones corporales dañadas de las bajas en este conflicto y atendidas por la Sanidad Militar española fueron predominantemente en extremidades (58\% y $85 \%$ respectivamente).

\section{Guerra del Golfo Pérsico (1990-91)}

Numerosos artículos analizan el tipo de baja en este conflicto. Destacamos como muestra 3 de ellos. Spalding ${ }^{15}$ describió que el $76 \%$ de las bajas atendidas (prisioneros) en un Hospital Militar británico tuvieron las lesiones en las extremidades.
La experiencia recogida por Pholmann ${ }^{16}$ de los heridos tratados en un Hospital de Arabia Saudí, fue similar al caso anterior, encontrándose que el $76 \%$ de las bajas presentaban lesiones en las extremidades, siendo más prevalentes en las inferiores que las superiores.

Finalmente, Souka ${ }^{17}$ analizó 361 bajas tratadas en un Hospital de Kuwait. Se observó en la serie un mayor número de lesiones en tórax y en abdomen que en conflictos previos.

\section{Guerra en Irak y Afganistán (2001-actualidad)}

En la Tabla 4 se exponen artículos anglosajones que muestran el área anatómica más afectada de las bajas estudiadas durante los conflictos en Irak y Afganistán.

Tras este análisis pormenorizado, podemos afirmar que las lesiones en miembros son las más comúnmente vistas durante los conflictos militares, siendo los miembros inferiores más afectados que los superiores.

En nuestro estudio, las áreas anatómicas más afectadas tanto por explosivo como por arma de fuego han sido los miembros inferiores y miembros superiores. Este hecho coincide con los datos obtenidos en la mayoría de las series. El resultado puede deberse a que la superficie corporal en las extremidades es superior al resto de regiones del organismo y a la utilización por parte de algunas bajas, de dispositivos de protección sobre la cabeza, el tórax y el abdomen.

Las bajas analizadas por los autores muestran que en todas las divisiones anatómicas, la mayor parte de las lesiones fueron provocadas por el explosivo. Además, debido al mecanismo de acción de este artefacto, la mayor parte de los heridos con dos o más áreas anatómicas dañadas habían sufrido ataque con IED.

La mayoría de las bajas de nuestra serie presentaron una única región anatómica afectada.

Tabla 4. Distribución por autores de las áreas más afectadas.

\begin{tabular}{|c|c|c|c|c|}
\hline Autor & Año & Guerra & $\mathbf{N}$ & Área más afectada \\
\hline Peoples ${ }^{18}$ & 2001-2002 & Afganistán & 224 & Extremidades inferiores $(58 \%)$ \\
\hline Fox $^{19}$ & 2001-2004 & Irak y Afganistán & 1524 & Extremidades (88\%) \\
\hline Owens ${ }^{20}$ & 2001-2005 & Irak y Afganistán & 1281 & Extremidades superiores (28\%) \\
\hline Beekley $^{21}$ & $2002-2003$ & Afganistán & 90 & Extremidades (44\%) \\
\hline Beitler $^{22}$ & $2002-2003$ & Afganistán & 204 & Extremidades inferiores (55\%) \\
\hline Hinsley $^{23}$ & 2003 & Irak & 482 & Extremidades $(82 \%)$ \\
\hline Zouris $^{24}$ & 2003 & Irak & 279 & Extremidades $(70 \%)$ \\
\hline Montgomery ${ }^{25}$ & 2003 & Irak & 294 & Extremidades inferiores $(25 \%)$ \\
\hline Patel $^{26}$ & 2003 & Irak & 154 & Extremidades $(56 \%)$ \\
\hline Reavley $^{27}$ & 2003-2004 & Irak & 66 & Extremidades (57\%) \\
\hline Colombo $^{28}$ & $2003-2007$ & Irak y Afganistán & 1499 & Cabeza $(28 \%)$ \\
\hline Gondusky ${ }^{29}$ & 2004 & Irak & 125 & Cabeza y cuello (53\%) \\
\hline Woodward ${ }^{30}$ & $2004-2007$ & Irak & 9289 & Extremidades (75\%) \\
\hline Murray $^{31}$ & 2003-2004 & Irak & 355 & Extremidades (41\%) \\
\hline Ramasamy $^{32}$ & 2006 & Irak & 104 & Extremidades (67\%) \\
\hline Owens $^{33}$ & $2001-2005$ & Irak y Afganistán & 1566 & Extremidades (54\%) \\
\hline Ramalingam $^{34}$ & 2003 & Irak & 124 & Extremidades $(55 \%)$ \\
\hline
\end{tabular}


En las extremidades, desde un punto de vista anatómico, las estructuras más afectadas por los proyectiles son los tejidos blandos, en segundo lugar el tejido óseo y por último los vasos sanguíneos y de los nervios ${ }^{35}$.

Las fracturas corticales pueden provocarse con proyectiles que mantengan una velocidad de $200 \mathrm{~m} / \mathrm{s}$. Un proyectil que choca contra el hueso, transfiere de forma máxima su energía cinética pudiendo desembocar en una gran lesión tisular. Además un proyectil de alta velocidad que provoca una cavidad temporal, puede causar un secuestro óseo e incluso una fractura indirecta, sospechada en trazos de fractura no desplazada y lineal (el 10\% de las fracturas en la Guerra de Vietnam eran de este tipo) ${ }^{11}$. Según estudios experimentales en animales, un proyectil cuya trayectoria se localice a $1 \mathrm{~cm}$ del periostio de un hueso largo puede motivar una fractura indirecta $^{36}$.

Comparados con los huesos, las arterias y las venas son mucho más elásticas y toleran mejor el fenómeno de cavitación. La tracción origina lesiones microscópicas como pérdidas de las células endoteliales, afectación de la membrana elástica interna y posibilidad de sangrado permanente ${ }^{37}$.

Al igual que los vasos sanguíneos, los nervios resisten considerablemente la tracción y empuje de la cavidad temporal. Así mismo se han demostrado lesiones histológicas en nervios periféricos secundarios a esta cavidad. Cirujanos militares han presenciado como pacientes tras sufrir una herida por arma de fuego sin evidencia de lesión macroscópica a nivel nervioso periférico y descartar motivo psicológico (stress de combate), muestran sintomatología de parestesias y paresias mantenidas en el tiempo ${ }^{35}$.

En el medio militar también debemos valorar las lesiones contusas provocadas por los mecanismos de desaceleración tras sufrir una explosión en un vehículo. Una serie soviética recogida durante el conflicto en Afganistán, indica que la distribución topográfica de las fracturas sufridas por los tripulantes soviéticos de vehículos blindados que sufrieron un ataque fue: miembro inferior $63 \%$, miembro superior $20,1 \%$, tórax y columna vertebral $13,5 \%$ y pelvis $2,8 \%{ }^{38}$.

\section{CONCLUSIONES}

En nuestro estudio, las áreas anatómicas más afectadas tanto por explosivo como por arma de fuego han sido los miembros inferiores y miembros superiores. Este hecho coincide con la conclusión obtenida en otras series.

En todas las divisiones anatómicas, el explosivo fue el agente causal de la mayor parte de las lesiones.

La mayoría de las bajas atendidas presentaron una única región anatómica afectada.

La mayor parte de los heridos con dos o más áreas anatómicas dañadas recibieron los impactos de un explosivo.

Es necesario por parte del Médico Militar el conocimiento de las características, manejo y tratamiento de las lesiones por arma de fuego y explosivos en las distintas regiones anatómicas.

\section{BIBLIOGRAFÍA}

1. Salas JH. Tratamiento de las heridas raquimedulares en la guerra. Rev Cubana Med Milit 1998;27(2): 120-23.
2. Carey ME. An analysis of US Army combat mortality and morbidity data. J Trauma. 1988;28(suppl):S515-S528

3. Homero. Canto IX. En: Homero. Iliada.1. ${ }^{a}$ ed. Barcelona: RBA Libros;2007. p. 235-262.

4. Fulgencio Martínez Saura. La Iliada y el Corpus Hipocraticum. Espacio, tiempo y forma, Serie II, Historia Antigua, t 9,1996,p. 169-193

5. Hipócrates. Sobre el médico. En: Hipócrates. Tratados hipocráticos. $1^{\mathrm{a}}$ Ed. Madrid: Alianza editorial;1996.p. 204-231.

6. Jenkins D, Dougherty P, Ryan J. Managing Ballistic Injury in the Military Environment: The concept of Foward Surgical Support. En Mahoney P, Ryan J, Brooks A, Schwab C, editors. Ballistic Trauma. $2^{\text {a }}$ ed. Springer;2004. 527-33.

7. Cutting P, Surgery in the Camp. En Mahoney P, Ryan J, Brooks A, Schwab C, editors. Ballistic Trauma. $2^{\text {a }}$ ed. Springer;2004. 585-91.

8. Palmer A. Survey of battle casualties, Eight Air Force, June, July and August 1944. En: Beyer JC. Wound Ballistic. Washington,DC:Department of the Army, Office of the Surgeon General;1962:573

9. Beebe GW, DeBakey JC. Battle casualties. Springfield,III. Clarles C Thomas; $1952: 92,186$

10. Baskin T, Holcomb J. Bombs, mines, blast, fragmentation and thermobaric mechanism of injury. En Mahoney P, Ryan J, Brooks A, Schwab C, editors. Ballistic Trauma. $2^{\mathrm{a}}$ ed. Springer;2004. 45-66.

11. Wound Data and Munitions Effectiveness Team. Evaluation of wound data and munition effectiveness in Vietnam (Final Report). Alexandria,VA: Defense Documentation Center of the Defense Logistics Agency.1970; In Vol. 3, table 4,p.C-7.

12. Jackson DS, Batty CG, Ryan JM, McGregor. Army Field Surgical Experience. The Falklands War. JR Army Med Corps 153(S1):44-7

13. Villanueva Serrano S, Martínez Pérez JM, Herrera Morillas F, Hernández-Abadía de Barbará A. Bajas por munición explosiva. Experiencia española en la antigua Yugoslavia. Med Mil.1997; 53(4): 339-43.

14. Villalonga Martínez L.M. Sanidad Militar en Bosnia-Herzegovina. Med Mil. 1996; 52(2): 177-80.

15. Spalding TJ, Stewart MP, Tulloch DN, Stephens KM. Penetrating missile injuries in the Gulf War 1991. Br J Surg 1991; 78(9): 1102-4.

16. Pohlmann,GP. War and medicine in the desert. A report of the $13^{\text {th }}$. Evacuation Hospital in Saudi Arabia. Wis Med J.1991 Sep;90(9):511-6

17. Souka HM. Management of Gulf War casualties. Br J Surg. 1992;79(12): 1307-8.

18. Peoples G, Gerlinger T, Craig R, Burlingame B. Combat casualties in Afghanistan cared for by a single Foward Surgical Team during the initial phases of Operation Enduring Freedom. Mil Med. 2005;170(6): 462-8.

19. Fox CJ, Gillespie DL, O'Donnell SD, Rasmussen TE, Goff JM, Jonson CA, etal. Contemporary management of wartime vascular trauma. J Vasc Surg, 2005;41(4): 638-44.

20. Owens BD, Kragh JF, Macaitis J, Svoboda SJ, Wenke JC. Characterization of extremity wounds in Operation Iraqi Freedom and Operation Enduring Freedom. J Orthop Trauma. 2007;21(4): 254-7.

21. Beekley A, Watts DM. Combat trauma experience with the United States Army 102nd Foward Surgical Team in Afghanistan. Am J Surg. 2004;187(5): 652-4.

22. Beitler A, Wortmann G, Hofmann L, Goff J. Operation Enduring Freedom: the 48th Combat Support Hospital in Afghanistan. Mil Med. 2006;171(3): 189-93.

23. Hinsley DE, Rosell PA, Rowlands TK, Clasper JC. Penetrating missile injuries during asymmetric warfare in the 2003 Gulf conflict. Br J Surg.2005;92(5): 637-42.

24. Zouris JM, Walter GJ, Dye J, Galarneau M. Wounding patterns for U.S. Marines and sailors during Operation Iraqi Freedom, major combat phase. Mil Med.2006; 171(3): 246-52.

25. Montgomery SP, Swiecki CW, Shriver CD. The evaluation of casualties from Operation Iraqi Freedom on return to the continental United States from March to June 2003. J Am Coll Surg. 2005; 201(1):7-12; discussion 12-3.

26. Patel TH, Wenner KA,Price SA, Weber MA, Leveridge A, McAtee SJ. US. Army Forward Surgical Team's experience in Operation Iraqi Freedom. J Trauma. 2004;57(2): 201-7.

27. Reavley P, Black J. Attendances at a Field Hospital emergency department during operations in Iraq November 2003 to March 2004 (Operation Telic III). J R Army Med Corp 2006; 152: 231-5.

28. Colombo CJ, Mount CA, Popa CA. Critical care medicine at Walter Reed Army Medical Center in support of the global war on terrorism. Crit Care Med.2008; 36(7 Supple): S388-94.

29. Gondusky J, Reiter M. Protecting Military Convoys in Iraq: An examination of Batlle Injuries Sustained by a Mechanized Battalion during Operation Iraqi Freedom II. Mil Med. 2005; 170(6): 546-9. 
Análisis de la topografía lesional en la baja de combate. Experiencia de la Sanidad Militar española...

30. Woodward EB, Clouse WD, Eliason JL, Peck MA, Bowser AN, Cox MW, etal Penetrating femoropopliteal injury during modern warfare: experience of Balad Vascular Registry. J Vasc Surg. 2008;47 (6): 1259-64.

31. Murray C, Reynolds J, Schroeder J, Harrison M, etal. Spectrum of care provided at an Echelon II Medical Unit during Operation Iraqi Freedom.Mil Med. 2005; 170(6):516-20.

32. Ramasamy A, Harrisson S, Lasrado I, Stewart MP. A review of casualties during the Iraqi insurgency 2006-A British field hospital experience. Injury.2009; 40(5):493-7.

33. Owens BD, Kragh JF, Wenke JC, Macaitis J, Wade CE, Holcomb JB. Combat wounds in operation Iraqi Freedom and operation enduring freedom. J Trauma. 2008; 64(2): 295-99.
34. Ramalingam T. Extremity injuries remain a high surgical workload in a conflict zone: experiences of a British Field Hospital in Iraq, 2003. J R Army Med Corp.2004; 150(3): 187-90.

35. Bellamy R, Zajtchuk R. The physics and biophysics of wound ballistics. En Conventional Warfare, ballistic, blast and burn injuries.1 ed. Textbook of Military Medicine Publications; 1991. 119-38.

36. Ragsdale, B. Experimental gunshot fractures. J. Trauma 1988;28 (1): S109-15.

37. Chen, D. The effect of high velocity missiles on adjacent blood vessels. J. Trauma 1990;6(2): S76-8

38. Burris D, etal. Weapons effects and parachute injuries. En Szul A. Emergency War Surgery. $3^{\mathrm{a}}$ ed. Borden Institute Walter Reed Medical Center; 2004.1.1-1.11 